\title{
Solution of Spinless Salpeter Equation with Generalized Hulthén Potential Using SUSYQM
}

\author{
A.N. IKOT ${ }^{a, *}$, C.N. IsonguYo ${ }^{b}$, Y.E. Chad-UMOREN ${ }^{a}$, And H. HASSANABAdi ${ }^{c}$ \\ ${ }^{a}$ Department of Physics, University of Port Harcourt, Choba, P.M.B 5323 Port Harcourt, Nigeria \\ ${ }^{b}$ Theoretical Physics Group, Department of Physics, University of Uyo, Nigeria \\ ${ }^{c}$ Department of Physics, University of Shahrood, Shahrood, Iran
}

(Received April 30, 2014)

The solution of spinless Salpeter equation with generalized Hulthén potential using SUSYQM formalism is presented. We obtained approximately the energy eigenvalues and the corresponding wave function in a closed form for any arbitrary $l$ state. We have also reported on the numerical result of our work.

DOI: 10.12693 /APhysPolA.127.674

PACS: 03.65.Ca, 03.65.Pm, 03.65.Db

\section{Introduction}

The spinless Salpeter equation (SSE) may be considered as a standard approximation to the BetheSalpeter formalism, neglecting the spin degree freedom [1]. The Bethe-Salpeter equation is the semirelativistic equation that describes the bound states of a two-body quantum field system in a relativistic covariant formalism [2]. In the recent times, there have been increasing interests in finding the analytical solutions of wave equations in relativistic and nonrelativistic quantum mechanics such as Schrödinger, Klein-Gordon, Dirac, Duffin Kemmer-Petiau (DKP) and spinless Bethe-Salpeter equations with different potential models [3-10]. The nonlocal nature of the Hamiltonian resulting from this approximation renders difficulty in obtaining SSE solutions [11]. The SSE is a generalization of Schrödinger equation in the quantum relativistic regime [12]. Consequently, many authors have resorted to approximate techniques to deal with the problems arising from the SSE [13]. In the recent times, many authors has investigated the SSE for various potential models. Hassanabadi et al. [14] studied the SSE with hyperbolic potential via the SUSYQM formalism. Zarrinkamar et al. [15] studied the two-body Sapelter equation with exponential potential using SUSYQM method.

In this work, we used supersymmetric quantum mechanics (SUSYQM) [16-18] to solve SSE with generalized Hulthén potential. The Hulthen potential is a shortrange potential in physics which behaves like a Coulomb potential for a small values of $r$ and decreases exponentially for a larger values of $r$ [19]. The Hulthén potential is very important in atom and molecule fields [20]. This potential has been used to explain the electronic properties of F-color centre in alkali halides [21].

\section{Two-body spinless Salpeter equation}

* corresponding author; e-mail: ndemikotphysics@gmail.com
The SSE for two-body particles interacting in a spherically symmetric potential in the centre of mass system appears as $[1,2]$,

$\left[\sum_{i=1,2}\left(\sqrt{-\nabla^{2}+m_{i}^{2}}-m_{i}\right)+V(r)-E_{n, l}\right] \chi(r)=0$,

where $\chi(r)=R_{n, l}(r) Y_{l, m}(\theta, \varphi)$. For heavy interacting particles and using appropriate transformation (see Refs. [2, 12]), one can recast SSE of Eq. (1) as [12]:

$\left[-\frac{\hbar^{2}}{2 \mu} \frac{\mathrm{d}^{2}}{\mathrm{~d} r^{2}}+\frac{l(l+1)}{2 \mu r^{2}}+W_{n l}(r)-\frac{W_{n l}^{2}(r)}{2 \tilde{m}}\right] \psi_{n l}(r)=0$,

where

$$
\begin{aligned}
& W_{n l}(r)=V(r)-E_{n l}, \\
& \mu=\frac{m_{1} m_{2}}{m_{1}+m_{2}}, \\
& \eta=\mu\left(\frac{m_{1} m_{2}}{m_{1} m_{2}-3 \mu^{2}}\right)^{\frac{1}{3}}, \\
& \tilde{m}=\frac{\eta^{3}}{\mu^{2}}=\frac{\left(m_{1} m_{2} \mu\right)}{\left(m_{1} m_{2}-3 \mu^{2}\right)} .
\end{aligned}
$$

We consider the generalized Hulthén potential defined as:

$$
V(r)=-V_{0}+\frac{V_{1}\left(A+B \mathrm{e}^{-\alpha r}\right)}{\left(C+D \mathrm{e}^{-\alpha r}\right)},
$$

where $V_{0}, V_{1}$ are the strength of the potential and $\alpha$ is the screening parameter. Substituting Eq. (7) into Eq. (2) yields

$$
\begin{gathered}
-\frac{\mathrm{d}^{2} \psi_{n l}}{\mathrm{~d} r^{2}}+\frac{1}{\hbar^{2}}\left\{\frac{l(l+1)}{r^{2}}+2 \mu\left[-V_{0}+V_{1}\left(\frac{A+B \mathrm{e}^{-\alpha r}}{C+D \mathrm{e}^{-\alpha r}}\right)-E_{n l}\right]\right. \\
-\frac{\mu}{\tilde{m}}\left(V_{0}+E_{n l}\right)^{2}+\frac{2 \mu V_{1} A}{\tilde{m}}\left(V_{0}+E_{n l}\right) \frac{1}{C+D \mathrm{e}^{-\alpha r}} \\
+\frac{2 \mu V_{1} B}{\tilde{m}}\left(V_{0}+E_{n l}\right) \frac{\mathrm{e}^{-\alpha r}}{C+D \mathrm{e}^{-\alpha r}}-\frac{V_{1}^{2} A^{2} \mu}{\tilde{m}} \\
\quad \times \frac{1}{\left(C+D \mathrm{e}^{-\alpha r}\right)^{2}}-\frac{2 \mu V_{1}^{2} B A}{\tilde{m}} \frac{e^{-\alpha r}}{\left(C+D e^{-\alpha r}\right)^{2}}
\end{gathered}
$$




$$
\left.-\frac{V_{1}^{2} B^{2} \mu}{\tilde{m}} \frac{\mathrm{e}^{-2 \alpha r}}{\left(C+D \mathrm{e}^{-\alpha r}\right)^{2}}\right\} \psi_{n l}(r)=0 .
$$

Equation (8) cannot be solved analytically because of the centrifugal term. Thus, we use the following approximation for the centrifugal term as [22]:

$$
\frac{1}{r^{2}}=\alpha^{2}\left(\frac{C}{C+D e^{-\alpha r}}\right)^{2},
$$

where $C=-D$ (see in Fig. 1). Substituting Eq. (9) into Eq. (8) yields

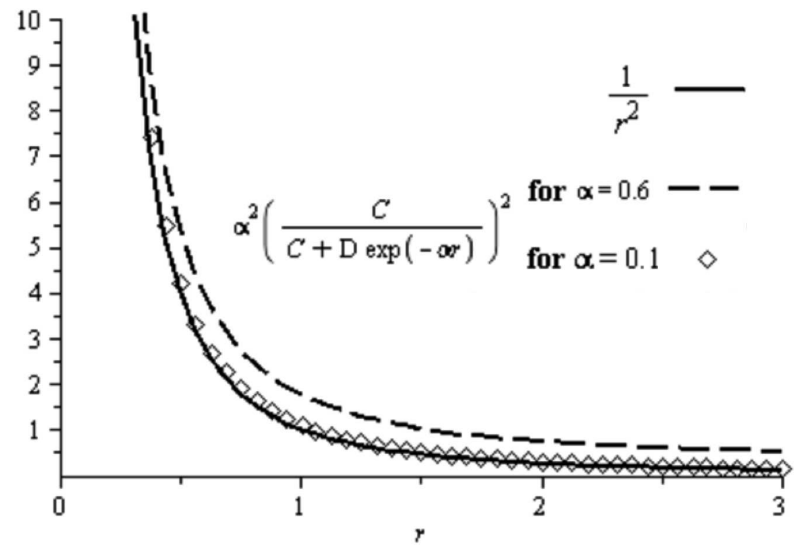

Fig. 1. $\frac{1}{r^{2}}$ and its approximation.

$$
\begin{aligned}
& -\frac{\mathrm{d}^{2} \psi_{n l}}{\mathrm{~d} r^{2}}+\frac{1}{\left(1+\frac{D \mathrm{e}^{-\alpha r}}{C}\right)^{2}}\left[\left(-\frac{\mu V_{1}^{2} B^{2}}{\tilde{m} \hbar^{2} C^{2}}\right.\right. \\
& \left.+\frac{2 \mu V_{1} B D}{\hbar^{2} C^{2}}+\frac{2 \mu V_{1} B D\left(V_{0}+E_{n l}\right)}{\hbar^{2} C^{2}}\right) \mathrm{e}^{-2 \alpha r} \\
& +\left(\frac{2 \mu V_{1} B}{\hbar^{2} C}+\frac{2 V_{1} \mu B\left(V_{0}+E_{n l}\right)}{\hbar^{2} C}+\frac{2 \mu V_{1} A D}{\hbar^{2} C^{2}}\right. \\
& \left.+\frac{2 \mu V_{1} A D\left(V_{0}+E_{n l}\right)}{\tilde{m} \hbar^{2} C^{2}}-\frac{2 \mu V_{1}^{2} A B}{\tilde{m} \hbar^{2} C^{2}}\right) \mathrm{e}^{-\alpha r} \\
& +\left(\frac{\alpha^{2} l(l+1)}{\hbar^{2}}+\frac{2 \mu V_{1} A}{\hbar^{2} C}+\frac{2 \mu V_{1} A\left(V_{0}+E_{n l}\right)}{\tilde{m} \hbar^{2} C}\right. \\
& \left.\left.-\frac{\mu A^{2} V_{1}^{2}}{\tilde{m} \hbar^{2} C^{2}}\right]\right] \psi_{n l}(r) \\
& =\frac{\mu}{\hbar^{2}}\left(\frac{\left(V_{0}+E_{n l}\right)^{2}}{\tilde{m}}+2\left(V_{0}+E_{n l}\right)\right) \psi_{n l}(r)
\end{aligned}
$$

or more explicitly, we write where

$$
-\frac{\mathrm{d}^{2} \psi_{n l}}{\mathrm{~d} r^{2}}+V_{\text {eff }}(r) \psi_{n l}(r)=\tilde{E} \psi_{n l}(r),
$$

$$
V_{\text {eff }}(r)=\frac{F \mathrm{e}^{-2 \alpha r}+G \mathrm{e}^{-\alpha r}+H}{\left(1+\frac{D \mathrm{e}^{-\alpha r}}{C}\right)^{2}},
$$

$$
\begin{aligned}
F & =\left(-\frac{\mu V_{1}^{2} B^{2}}{\tilde{m} \hbar^{2} C^{2}}+\frac{2 \mu V_{1} B D}{\hbar^{2} C^{2}}\right. \\
& \left.+\frac{2 \mu V_{1} B D\left(V_{0}+E_{n l}\right)}{\tilde{m} \hbar^{2} C^{2}}\right), \\
G & =\left(\frac{2 \mu V_{1} B}{\hbar^{2} C}+\frac{2 V_{1} \mu B\left(V_{0}+E_{n l}\right)}{\tilde{m} \hbar^{2} C}+\frac{2 \mu V_{1} A D}{\hbar^{2} C^{2}}+\right. \\
& \left.\frac{2 \mu V_{1} A D\left(V_{0}+E_{n l}\right)}{\tilde{m} \hbar^{2} C^{2}}-\frac{2 \mu V_{1}^{2} A B}{\tilde{m} \hbar^{2} C^{2}}\right), \\
H & =\left(\frac{\alpha^{2} l(l+1)}{\hbar^{2}}+\frac{2 \mu V_{1} A}{\hbar^{2} C}+\frac{2 \mu V_{1} A\left(V_{0}+E_{n l}\right)}{\tilde{m} \hbar^{2} C}\right. \\
& \left.-\frac{\mu A^{2} V_{1}^{2}}{\tilde{m} \hbar^{2} C^{2}}\right), \\
\tilde{E}_{n l} & =\frac{\mu}{\hbar^{2}}\left(\frac{\left(V_{0}+E_{n l}\right)^{2}}{\tilde{m}}+2\left(V_{0}+E_{n l}\right)\right) .
\end{aligned}
$$

In order to solve Eq. (11), we have to first solve the associated Riccati equation

$$
W^{2}(r) \mp W^{\prime}(r)=V_{\text {eff }}(r)-\tilde{E}_{0, l},
$$

for which we propose a solution of the form

$$
W(r)=\frac{f \mathrm{e}^{-\alpha r}}{\left(1+\frac{D \mathrm{e}^{-\alpha r}}{C}\right)}+q .
$$

Substituting Eqs. (12) and (18) into the Riccati equation, we obtain

$$
\begin{aligned}
& \frac{f^{2} \mathrm{e}^{-2 \alpha r}}{\left(1+\frac{D \mathrm{e}^{-\alpha r}}{C}\right)^{2}}+(q)^{2}+\frac{2 f q \mathrm{e}^{-\alpha r}}{\left(1+\frac{D \mathrm{e}^{-\alpha r}}{C}\right)}+\frac{\alpha f \mathrm{e}^{-\alpha r}}{\left(1+\frac{D \mathrm{e}^{-\alpha r}}{C}\right)^{2}}= \\
& \frac{F \mathrm{e}^{-2 \alpha r}+G \mathrm{e}^{-\alpha r}+H}{\left(1+\frac{D \mathrm{e}^{-\alpha r}}{C}\right)^{2}}-\tilde{E}_{0, l}
\end{aligned}
$$

or solving Eq. (19) completely, we obtain the following three sets relationship:

$$
\begin{aligned}
& \tilde{E}_{0, l}=-(q)^{2}+H, \\
& f=\frac{\frac{D \alpha}{C} \pm \sqrt{\left(\frac{D \alpha}{C}\right)^{2}+4\left(F+\frac{D^{2} H}{C^{2}}-\frac{D G}{C}\right)}}{2}, \\
& q=\frac{\left(-f^{2}+F-\frac{D^{2} H}{C^{2}}\right)}{2 f \frac{D}{C}} .
\end{aligned}
$$

Now based on Eq. (A.2), we can obtain the supersymmetric partner potentials as

$$
\begin{gathered}
V_{+}(r)=-\frac{C}{D} \frac{f\left(f+\frac{D \alpha}{C}\right) \mathrm{e}^{-\alpha r}}{\left(1+\frac{D e^{-\alpha r}}{C}\right)^{2}} \\
+\frac{\left(\frac{C}{D} F-\frac{D}{C} H\right) \mathrm{e}^{-\alpha r}}{\left(1+\frac{D \mathrm{e}^{-\alpha r}}{C}\right)}+(q)^{2}, \\
\left.V_{-} r\right)=-\frac{C}{D} \frac{f\left(f-\frac{D \alpha}{C}\right) \mathrm{e}^{-\alpha r}}{\left(1+\frac{D \mathrm{e}^{-\alpha r}}{C}\right)^{2}}
\end{gathered}
$$




$$
+\frac{\left(\frac{C}{D} F-\frac{D}{C} H\right) \mathrm{e}^{-\alpha r}}{\left(1+\frac{D \mathrm{e}^{-\alpha r}}{C}\right)}+(q)^{2} .
$$

Therefore, it is shown that $V_{+}(r)$ and $V_{-}(r)$ are shape invariant, satisfying the shape-invariant condition

$$
V_{+}\left(r, a_{0}\right)=V_{-}\left(r, a_{1}\right)+R\left(a_{1}\right),
$$

with $a_{0}=f$ and $a_{i}$ is a function of $a_{0}$, i.e $a_{1}=f\left(a_{0}\right)=$ $a_{0}+\frac{D \alpha}{C}$. Therefore, $a_{n}=f\left(a_{0}\right)=a_{0}+\left(\frac{D \alpha n}{C}\right)$. Thus, we can see that the shape invariance holds via a mapping of the form $f \rightarrow f+\left(\frac{D \alpha}{C}\right)$. From Eq. (A.5), we have

$R\left(a_{1}\right)=\left(\frac{\left(-a_{0}^{2}+F-\frac{D^{2} H}{C^{2}}\right)}{2 a_{0} \frac{D}{C}}\right)^{2}-\left(\frac{\left(-a_{1}^{2}+F-\frac{D^{2} H}{C^{2}}\right)}{2 a_{1} \frac{D}{C}}\right)^{2}$,
$R\left(a_{2}\right)=\left(\frac{\left(-a_{1}^{2}+F-\frac{D^{2} H}{C^{2}}\right)}{2 a_{1} \frac{D}{C}}\right)^{2}-\left(\frac{\left(-a_{2}^{2}+F-\frac{D^{2} H}{C^{2}}\right)}{2 a_{2} \frac{D}{C}}\right)^{2}$,

$R\left(a_{n}\right)=\left(\frac{\left(-a_{n-1}^{2}+F-\frac{D^{2} H}{C^{2}}\right)}{2 a_{n-1} \frac{D}{C}}\right)^{2}-\left(\frac{\left(-a_{n}^{2}+F-\frac{D^{2} H}{C^{2}}\right)}{2 a_{n} \frac{D}{C}}\right)^{2}$

The energy eigenvalues can be obtained as follows

$$
\tilde{E}_{n l}=\tilde{E}_{n l}^{-}+\tilde{E}_{0, l},
$$

where

$$
\begin{aligned}
\tilde{E}_{n l}^{-} & =\sum_{k=1}^{n} R\left(a_{k}\right)=\left(\frac{\left(-a_{0}^{2}+F-\frac{D^{2} H}{C^{2}}\right)}{2 a_{0} \frac{D}{C}}\right)^{2} \\
& -\left(\frac{\left(-a_{n}^{2}+F-\frac{D^{2} H}{C^{2}}\right)}{2 a_{n} \frac{D}{C}}\right)^{2} .
\end{aligned}
$$

By substituting Eqs. (22) and (27) into Eq. (26), we have

$$
\tilde{E}_{n l}=H-\left(\frac{\left(-a_{n}^{2}+F-\frac{D^{2} H}{C^{2}}\right)}{2 a_{n} \frac{D}{C}}\right)^{2} .
$$

Substituting Eq. (16) into Eq. (28), we obtain the energy equation for the generalized Hulthén potential as

$$
\begin{gathered}
\frac{\mu}{\hbar^{2}}\left(\frac{\left(V_{0}+E_{n l}\right)^{2}}{\tilde{m}}+2\left(V_{0}+E_{n l}\right)\right)= \\
H-\left(\frac{\left(-a_{n}^{2}+F-\frac{D^{2} H}{C^{2}}\right)}{2 a_{n} \frac{D}{C}}\right)^{2} .
\end{gathered}
$$

Defining a new variable of the form $s=-\frac{D}{C} \mathrm{e}^{-\alpha r}$, and substituting it into Eq. (11), we have

$$
\begin{aligned}
& s^{2} \frac{\mathrm{d}^{2} \psi_{n, J}(s)}{\mathrm{d} s^{2}}+s \frac{\mathrm{d} \psi_{n, J}(s)}{\mathrm{d} s}+\frac{1}{(1-s)^{2}} \\
& \quad \times\left(-\omega_{1} s^{2}+\omega_{2} s-\omega_{3}\right) \psi_{n, J}(s)=0,
\end{aligned}
$$

$$
\begin{aligned}
& \omega_{1}=\frac{1}{\alpha^{2}}\left(-\tilde{E}_{n, l}+\frac{C^{2}}{D^{2}} F\right), \\
& \omega_{2}=-\frac{1}{\alpha^{2}}\left(2 \tilde{E}_{n, l}-\frac{C}{D} G\right), \\
& \omega_{3}=-\frac{1}{\alpha^{2}}\left(\tilde{E}_{n, l}-H\right) .
\end{aligned}
$$

The corresponding wave function can be determined from Eq. (30) as

$$
\begin{gathered}
\psi_{n, l}(r)=N_{n l}\left(-\frac{D}{C} \mathrm{e}^{-\alpha r}\right)^{\sqrt{\omega_{3}}}\left(1+\frac{D}{C} \mathrm{e}^{-\alpha r}\right)^{\frac{1}{2}+\sqrt{\omega_{1}-\omega_{2}+\omega_{3}+\frac{1}{4}}} \\
\times P_{n}^{\left(2 \sqrt{\omega_{3}}, 2 \sqrt{\omega_{1}-\omega_{2}+\omega_{3}+\frac{1}{4}}\right)}\left(1+\frac{2 D}{C} \mathrm{e}^{-\alpha r}\right) .
\end{gathered}
$$

The energy of the system for different states is reported in Table. In Fig. 2, we have also presented the wave function of $|1,0\rangle,|2,0\rangle$ and $|3,0\rangle$ states. The behavior of the energy of the system is plotted in Fig. 3. It is clear that as $\alpha$ increases the energy of the system increases.

TABLE

The energy of the system for different states and $m_{1}=m_{2}=1, \alpha=0.1, V_{0}=0.5, V_{1}=-1.5$, $A=-2, B=2, C=-1, D=1$.

\begin{tabular}{c|c|c|c}
\hline \hline$|n, l\rangle$ & $E_{n, l}$ & $|n, l\rangle$ & $E_{n, l}$ \\
\hline$|1,0\rangle$ & -7.489974874 & $|3,0\rangle$ & -7.459591794 \\
$|1,1\rangle$ & -7.486342256 & $|3,1\rangle$ & -7.446381258 \\
$|1,2\rangle$ & -7.484313483 & $|3,2\rangle$ & -7.436491673 \\
$|1,3\rangle$ & -7.483027988 & $|3,3\rangle$ & -7.428888863 \\
$|1,4\rangle$ & -7.482142499 & $|3,4\rangle$ & -7.422888452 \\
$|2,0\rangle$ & -7.477371993 & $|4,0\rangle$ & -7.436491673 \\
$|2,1\rangle$ & -7.469136867 & $|4,1\rangle$ & -7.418042984 \\
$|2,2\rangle$ & -7.463568181 & $|4,2\rangle$ & -7.403326840 \\
$|2,3\rangle$ & -7.459591794 & $|4,3\rangle$ & -7.391428032 \\
$|2,4\rangle$ & -7.456621412 & $|4,4\rangle$ & -7.381652806
\end{tabular}

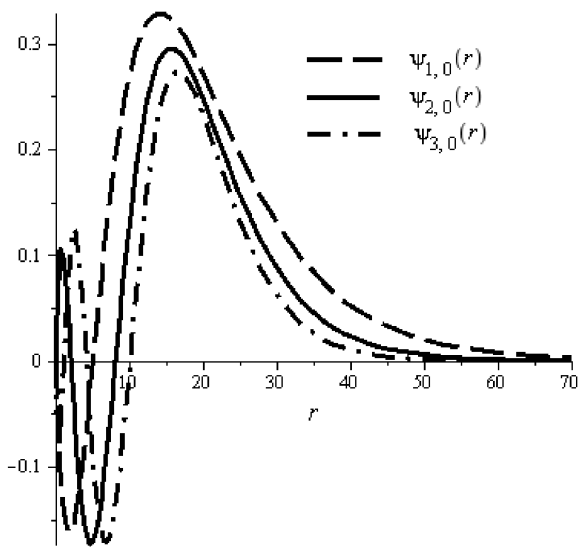

Fig. 2. The wave function of the system for different states.

where 


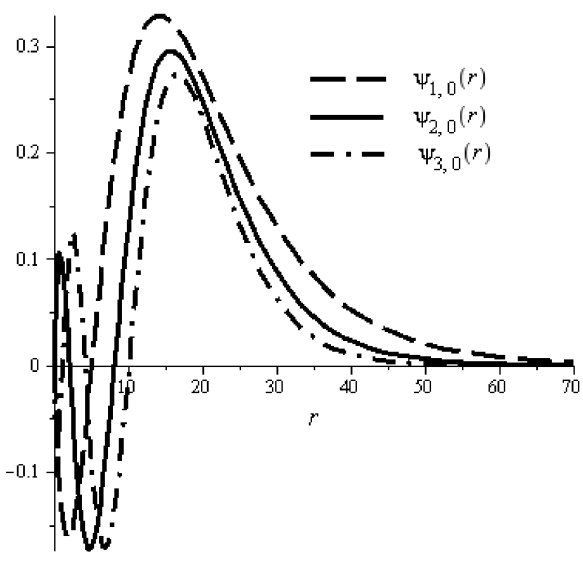

Fig. 3. The behavior of the energy of the system for different states.

\section{Conclusion}

In this work, we solve the two-body spinless Salpeter equation with generalized Hulthén potential with proper approximation to the centrifugal term using the powerful SUSQM technique. We obtain explicitly, the bound state energy eigenvalues and the corresponding wave function in a closed form. Finally, we computed the energy eigenvalues of our work numerically in order to check the accuracy of our results.

\section{Appendix A}

\section{Supersymmetry quantum mechanics}

In the SUSYQM we normally deal with the partner Hamiltonians [17-19]:

$$
H_{ \pm}=\frac{p^{2}}{2 m}+V_{ \pm}(x)
$$

where

$$
V_{ \pm}(x)=\Phi^{2}(x) \pm \Phi^{\prime}(x) .
$$

In the case of good SUSY, i.e. $E_{0}=0$, the ground state of the system is obtained via

$$
\varphi_{0}^{-}(x)=C \mathrm{e}^{-U},
$$

where $C$ is a normalization constant and

$$
U(x)=\int_{x_{0}}^{x} \mathrm{~d} z \Phi(z) .
$$

Next, if the shape invariant condition

$$
V_{+}\left(a_{0}, x\right)=V_{-}\left(a_{1}, x\right)+R\left(a_{1}\right),
$$

where $a_{1}$ is a new set of parameters uniquely determined from the old set $a_{0}$ via the mapping $F: a_{0} \mapsto a_{1}=F\left(a_{0}\right)$ and $R\left(a_{1}\right)$ does not include $x$, the higher state solutions are obtained via

$$
\begin{aligned}
& E_{n}=\sum_{s=1}^{n} R\left(a_{s}\right), \\
& \varphi_{n}^{-}\left(a_{0}, x\right)=\prod_{s=0}^{n-1}\left(\frac{A^{\dagger}\left(a_{s}\right)}{\left[E_{n}-E_{s}\right]^{1 / 2}}\right) \varphi_{0}^{-}\left(a_{n}, x\right), \\
& \varphi_{0}^{-}\left(a_{n}, x\right)=C \exp \left(-\int_{0}^{x} \mathrm{~d} z \Phi\left(a_{n}, z\right)\right),
\end{aligned}
$$

$$
\begin{aligned}
& \text { where } \\
& A_{s}^{\dagger}=-\frac{\partial}{\partial x}+\Phi\left(a_{s}, x\right) .
\end{aligned}
$$

Therefore, this condition determines the spectrum of the bound states of the Hamiltonian

$$
H_{s}=-\frac{\partial^{2}}{\partial x^{2}}+V_{-}\left(a_{s}, x\right)+E_{s} .
$$

and the energy eigenfunctions of

$$
H_{s} \varphi_{n-s}^{-}\left(a_{s}, x\right)=E_{n} \varphi_{n-s}^{-}\left(a_{s}, x\right), \quad n \geq s
$$

are related via

$$
\varphi_{n-s}^{-}\left(a_{s}, x\right)=\frac{A^{\dagger}}{\left[E_{n}-E_{s}\right]^{1 / 2}} \varphi_{n-(s+1)}^{-}\left(a_{s+1}, x\right) .
$$

\section{References}

[1] G.C. Wick, Phys. Rev. 96, 1124 (1954).

[2] E.E. Salpeter, H.A. Bethe, Phys. Rev. 84, 1232 (1985).

[3] L. Chang, C.D. Roberts, Phys. Rev. Lett. 103, 081601 (2009).

[4] H. Hassanabadi, B.H. Yazarloo, S. Zarrinkamar, A.A Rajabi, Phys. Rev. C 84, 064003 (2011).

[5] W. Lucha, F.F. Schoberl, Int. J. Mod. Phys. A 17, 2233 (2002)

[6] R. Hall, W. Lucha, Int. J. Mod. Phys. A 22, 1899 (2007).

[7] R. Hall, W. Lucha, F.F. Schoberl, Int. J. Mod. Phys. A 17, 1931 (2002).

[8] R. Hall, W. Lucha, F.F. Schoberl, Int. J. Mod. Phys. A 18, 2657 (2003).

[9] P. Maris, C.D. Roberts, Int. J. Mod. Phys. E 12, 297 (2003)

[10] P. Maris, C.D. Roberts, Phys. Rev. C 56, 3369 (1997).

[11] W. Lucha, F.F. Schoberl, Phys. Rev. D 50, 5443 (1994).

[12] S. Hassanabadi, M. Ghominejad, S. Zarrinkamar, H. Hassanabadi, Chin. Phys. B 22, 060303 (2013).

[13] W. Lucha, F.F. Schoberl, D. Gromes, Phys. Rep. 200, 127 (1991).

[14] H. Hassanabadi, S. Zarrinkamar, B.H. Yazarloo, Chin. J. Phys. 50, 783 (2012).

[15] S. Zarrinkamar, A.A. Rajabi, H. Hassanabadi, FewBody Syst 52, 165 (2012).

[16] F. Cooper, A. Khare, U. Sukhatme, Phys. Rep. 251, 267 (1995).

[17] B. Bagchi, Supersymmetry in Quantum and Classical Mechanics, Chapman and Hall/CRC, New York 2000.

[18] G. Junker, Supersymmetric Methods in Quantum and Statistical Physics, Springer-Verlag, Berlin 1996.

[19] S. Zarrinkamar, A.A. Rajabi, H. Hassanabadi, Phys. Scr. 84, 065008 (2011).

[20] S. Flugge, Practical Quantum Mechanics, Springer, Berlin 1974

[21] F. Dominguez-Adame, Phys. Lett. B 136, 175 (1989).

[22] B.H. Yazarloo, H. Hassanabadi, S. Zarrinkamar, Eur. Phys. J. Plus 127, 51 (2012). 